\title{
FULLY-AUTOMATED LIQUID PENETRANT INSPECTION LINE SIMULATION MODEL FOR INCREASING PRODUCTIVITY
}

\author{
Popescu, D.; Anania, F. D.; Cotet, C. E. \& Amza, C. G. \\ University Politehnica of Bucharest, IMST Faculty, \\ 313, Splaiul Independentei, Sector 6, 060042, Bucharest, Romania \\ E-Mail: diana@mix.mmi.pub.ro
}

\begin{abstract}
By proposing an optimization model for a new automated liquid penetrant inspection (LPI) system in order to increase its productivity, the paper tries to identify the best algorithm to solve this case study. The architecture of the system is dictated by the successive stages of the inspection process and the available conditions in the work shop. As a novelty in the field, the authors developed such a fully automated LPI system for inspecting different parts, which eliminates the need of the visual inspection made by operator, using instead dedicated software solution for processing the digital images of the inspected parts and for giving the pass/fail verdict. In the present case study, the attention was focused on optimizing the new LPI system architecture. Simulations in different working scenarios are run with the purpose to increase productivity by optimizing the critical waiting times within the system and by establishing the best order for inspecting parts belonging to three families subjected to LPI method. Moreover, the results of the simulation are used for programming the system by setting the optimal values of the functional parameters of system's equipment in order to avoid running a large number of tests which are expensive and time consuming.

(Received in April 2012, accepted in February 2013. This paper was with the authors 3 months for 4 revisions.)
\end{abstract}

Key Words: Discrete Event Simulation, LPI Line Design, Productivity, Optimization

\section{INTRODUCTION}

\subsection{Problem statement}

Liquid penetrant inspection (LPI) is a non-destructive evaluation (NDE) method used for verifying the presence of open discontinuities at the surface of analysed parts submitted for inspection [1,2]. Currently the evaluation is visually performed by an inspector [3] in standard conditions [4-6], the results being influenced by subjective issues such as experience, knowledge and motivation. Furthermore, the pass/fail grade for the inspected part depends on the size, shape and orientation of the flaws, taking into consideration also the fact that the indications (visible in colour) are larger than the actual defect [7]. Therefore, the automation of this process provides advantages over the total inspection time/cost, by also increasing its reliability, and represents an important research subject in the field. However, the difficulties of automating this process relates not only to the evaluation process itself (which must be performed with dedicated software in order to automatically process the images acquired using a digital camera), but also to the determination and control of the process parameters such as dwell time, developer time, drying time, quantity of penetrant, developer and cleaning water, pressure for spraying solutions with penetrant, developer and cleaning water, transportation speed or distance between stations [8]. Moreover, as it is the case of the LPI system discussed in this case study, usually more than one type of part is inspected, thus the 
determination process of the optimal order in which pieces are inspected is mandatory for ensuring a good productivity of the system.

In this context, the current paper proposes an optimization model for a new fully automated LPI process, its functioning being simulated in different working scenarios with the purpose to increase the productivity. The waiting times for dwell, dry and developer operations have significant larger values compared to other process times within the system. They were identified as critical and considered for the optimisation study. Thus, the model, developed using a commercial simulation software package (Delmia Quest, Dassault Systemes, France), works by optimizing these waiting times and by establishing the optimal parts inspection order of parts belonging to three different types, and contributes to the reduction of the number of experiments required for determining the optimal values for LPI process parameters - a time consuming and expensive task.

The simulation of the LPI system under research is extremely useful in designing and programming the system because it allows the early identification of the potential flow concentrators which generate low productivity or even blockage $[9,10]$. Flow concentrators also generate underutilization of the structural elements of the inspection system leading to financial loss [11]. Thus, as main novelty of the research, the data obtained from the simulation of the LPI system functioning with the optimal configuration is used for generating commands for different equipment such as buffers and nozzles for spraying penetrant liquid, water and developer, as it will be presented at the end of section 4 of the present study.

Next section outlines research in which computer simulations performed with different commercial software packages are used for designing and optimizing automated systems. Section 2 describes the preliminary architecture of an automated LPI system currently under research, the process times required by each inspected part, details of the inspection process steps and the research approach taken. Section 3 presents the model used for simulation and the input parameters, and defines the simulation analysis cases and working scenarios. Section 4 analysis the simulation results and the productivity for each case in order to establish the best waiting times distribution within the system and the optimal parts' inspection order. Conclusions are presented in section 5.

\subsection{Literature review}

Determining the best design of an automated manufacturing system, assembly line or inspection system and optimizing their functioning represents a research subject in the engineering field with important practical applications. One of the best tools for achieving these purposes are the computer simulations used to analyse productivity in different working scenarios, to see and optimize all the production phases, to identify possible bottlenecks where the material flow is slowed or blocked, to determine the parts optimal manufacturing order or to perform production line balancing.

Our study takes the above mentioned approach, so that the LPI system functioning is being simulated as a discrete material flow [12] with the purpose to determine an optimal configuration of the system that in this case means to optimize the distributions of waiting times within the system and to establish the best order for pulling the parts from buffers. The other elements of the system (such as conveyor length, distance between working stations, operation times) are imposed by the pre-existent conditions in the working shop. The system is modelled as containing working stations, transportation lines and buffers as structural elements.

The literature survey was focused on similar case studies in which commercial simulation software packages are used for optimizing automated production systems for the increase of their productivity and machines utilization. However, no computer optimization based on 
material flow simulation was made for increasing the productivity of automated LPI systems, or any command and programming of these systems were performed based on such simulation results.

Masood [13] refers to an automated production line for automotive cylinder blocks. The simulation, performed using Simul8 software, considers modifying the sequencing of the operations for which the cycle times are the largest in order to reduce the total time and increase the machine utilization. The process starts with the identification of the critical times for each operation and machine. Every station has a fixed cycle time, a certain number of operations and the transportation time between working stations is constant. The optimization consisted in analysing the times and re-sequencing the cutting tool operations, which led to $32 \%$ reduction of cycle times and $65 \%$ increase in productivity.

A simulation study of a manufacturing cell is presented in [14] with the purpose of examining the impact over the throughput time of different modifications in the buffer size, product volumes or product sequence. Longo et al. [15] presents the use of modelling, simulations and ergonomic analyses tools for the effective design of an assembly line for producing heaters. The system consisting in four working stations and two transportation lines is simulated using eM-Workplace from Tecnomatix Technologies, process and transportation times, operations frequencies for each station etc. being input by user in the model.

The use of simulation as a method to establish the layout and process times of automated lines and to solve technological design problems is also presented in [16] for inspecting specular surfaces and in [17] for optimizing machining sequences within a manufacturing system using Delmia software. Furthermore, Ekren et al. presented in [18] a simulation model performed using Arena software for analysing two different type of plant layouts, to evaluate the average flow time through the system and to study the influence of machines positions, batch sizes, transporter capacities etc. on the processing times.

Nguyen [19] presents the application of simulation modelling to manufacturing line design in Japanese plants before the actual purchasing and implementation of machine, robots, transport or transfer systems, etc. The paper is developing a general framework based on a linear programming model for selecting different alternatives for simulation in order to design a new manufacturing line or modify an existing line. Arena was used to simulate different alternatives of manufacturing line in order to determine the one that provides the highest productivity and resources utilization and the smallest manufacturing costs.

\section{PRELIMINARY ARCHITECTURE OF THE IN-LINE AUTOMATED LIQUID PENETRANT INSPECTION SYSTEM}

A typical in-line set-up for LPI consists of the following work stations (WS): 1. penetrant application, 2. surface penetrant removal, 3. drying penetrant liquid, 4. developer application and 5. inspection, the operations performed in these WS being illustrated in Fig. 1. According to these standard operations and to the objective conditions in the working shop, a preliminary architecture of the in-line automated LPI system under research was established and it is presented in Fig. 2. Three types of parts $(P 1, P 2, P 3)$ are inspected using this system, the difference between them being given by their dimensions, materials and manufacturing technology (welded parts, casting parts and machined parts), therefore different types of defects should be detected by the LPI system.

Table I presents the process times for each operation and family of parts. Parts are supplied to the automated LPI in-line system from three buffers (one for each parts family) and after inspection (based on the image analysis and on the criteria implemented in a defects knowledge database) a decision is taken regarding acceptance or rejection. The values presented in Table I are established by practice, according to the standards and regulations in 
force, considering the type of penetrant and developer used and the type of defects to inspect.

The dwell time is defined as the time for the liquid penetrant to infuse into the material's surface. The developer time is the time for a reliable image to appear on the part surface.

The parts are cleaned before their placement in buffers; therefore the first stage of the LPI process included in automation is the penetrant application by spraying. Afterwards the liquid in excess is eliminated from the surface, parts' washing being an important stage of the inspection process. The water pressure, duration and temperature must be carefully considered in order not to over-wash the part and therefore to lose the indications. According to standards, the water pressure shall not exceed $345 \mathrm{~Pa}$, the water temperature should be less than $43^{\circ} \mathrm{C}$, and the angle for spraying the water is $45^{\circ} \mathrm{C}$ to the part surface [4].

The next step in the process consists in applying a developer, usually a white dried powder, which penetrates in the cracks presented on the surface part (or which communicates with the surface), by reverse capillary action, and produces indications on the surface (Fig. 1).

The cleaning process is followed by drying in order to eliminate the cleaning solution from the part for allowing the developer to penetrate into the discontinuities. As drying methods, hot air, drying lamps or drying oven can be used.

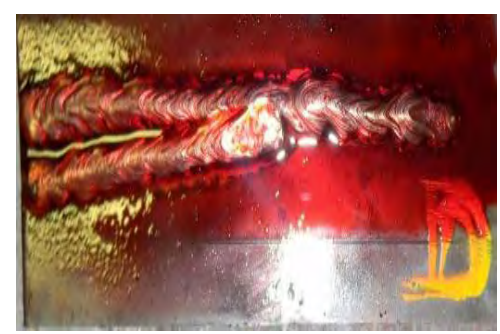

a)

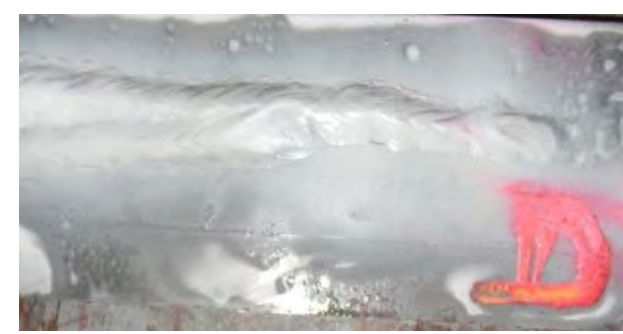

b)

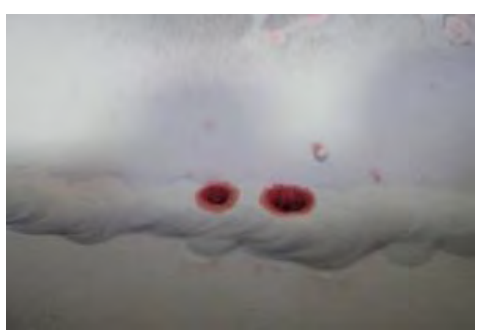

c)

Figure 1: Example of successive operations of a LPI process for a welded part: a) penetrant application, b) developer application, c) indication of defects presence in the weld.

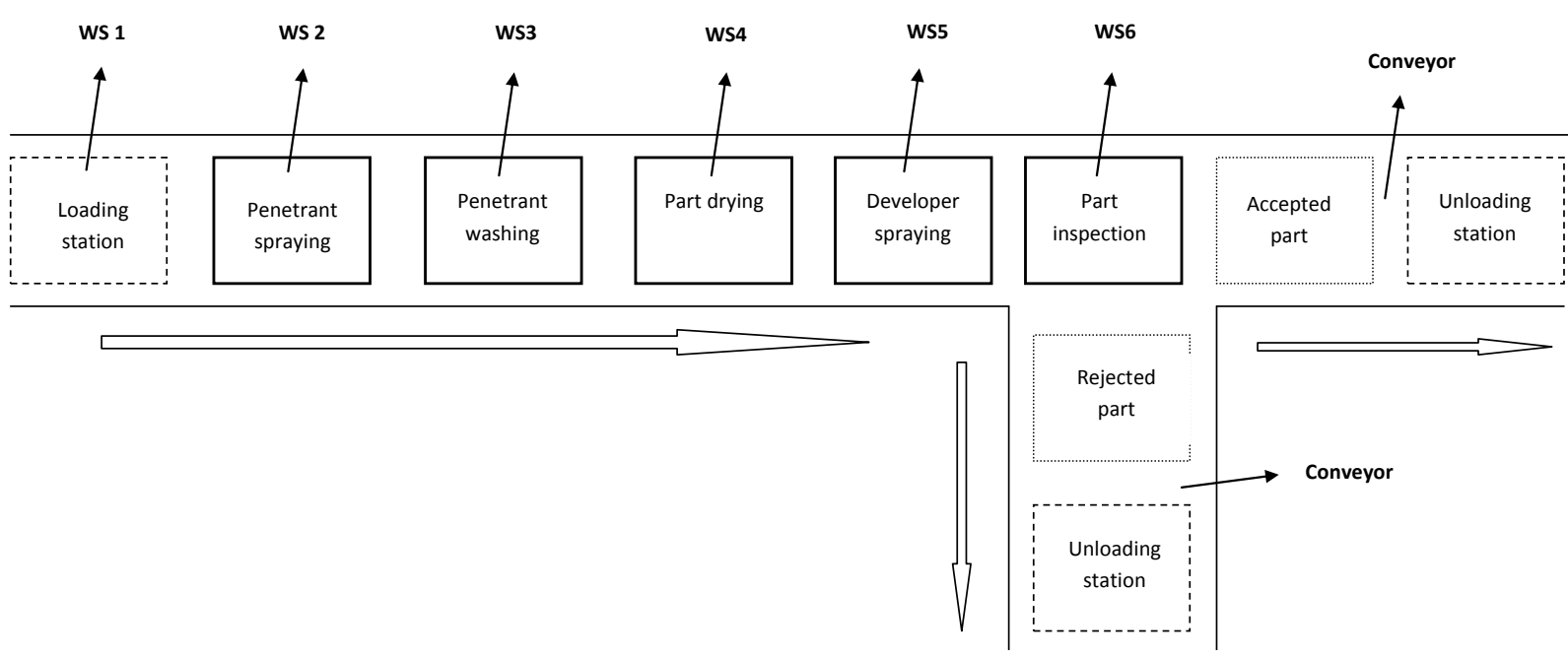

Figure 2: Fully-automated in-line automated liquid penetrant inspection system.

An analysis of the commercial LPI systems [20-25] showed that the automation of the process is related only to the parts transportation between stations and their positioning in each station working space. Several characteristics of these commercial automated inspection lines are further emphasized:

- Parts transportation between posts is made using a conveyor, a gripper or a combination between these two. 
- Spraying or immersing is used for applying the penetrant.

- Parts are dried by using a hot air or a lamp.

- The developer is applied by immersing or spraying.

Table I: LPI process times for three families of parts for each working station.

\begin{tabular}{|l|c|c|c|}
\hline Part/defect/manufacturing process & $\begin{array}{c}\text { P1/porosity/ } \\
\text { castings }\end{array}$ & $\begin{array}{c}\text { P2/cracks/ } \\
\text { machining }\end{array}$ & $\begin{array}{c}\text { P3/overlap, underfill, } \\
\text { undercut/welding }\end{array}$ \\
\hline Penetrant spraying time, WS2 & $10 \mathrm{~s}$ & $10 \mathrm{~s}$ & $10 \mathrm{~s}$ \\
\hline $\begin{array}{l}\text { Dwell time - in WS2, WS3 and/or } \\
\text { between WS2 and WS3 }\end{array}$ & $350 \mathrm{~s}$ & $330 \mathrm{~s}$ & $300 \mathrm{~s}$ \\
\hline Penetrant washing time, WS3 & $10 \mathrm{~s}$ & $15 \mathrm{~s}$ & $20 \mathrm{~s}$ \\
\hline Drying time, WS4 & $200 \mathrm{~s}$ & $250 \mathrm{~s}$ & $350 \mathrm{~s}$ \\
\hline Developer spraying time, & $7 \mathrm{~s}$ & $10 \mathrm{~s}$ & $12 \mathrm{~s}$ \\
\hline $\begin{array}{l}\text { Developer time - in WS5, WS6 and/or } \\
\text { between WS5 and WS6 }\end{array}$ & $600 \mathrm{~s}$ & $500 \mathrm{~s}$ & $400 \mathrm{~s}$ \\
\hline Inspection time, WS6 & $40 \mathrm{~s}$ & $30 \mathrm{~s}$ & $50 \mathrm{~s}$ \\
\hline
\end{tabular}

Moreover, in all these LPI systems the inspection is made visually by a human inspector and, although, patents [26-27] presents several approaches and general frameworks which include also automatic image processing of the flaws, to the best of authors' knowledge, these equipment are not yet implemented, nor an application of simulation modelling in designing these inspection systems was found in the literature.

Therefore, as a novelty for the LPI field, this research developed a fully automated LPI system for inspecting different parts that eliminates the need of the visual inspection made by the operator. It proposes to use a dedicated software solution for processing the digital images of the inspected parts and for giving the pass/fail verdict. In the present case study, the attention was focused on optimizing the new LPI system architecture and thus, a simulation model made in Delmia Quest was used for increasing productivity by balancing the waiting times for dwell, drying and developer operations between WS, and for establishing the best inspection order for the three different types of parts.

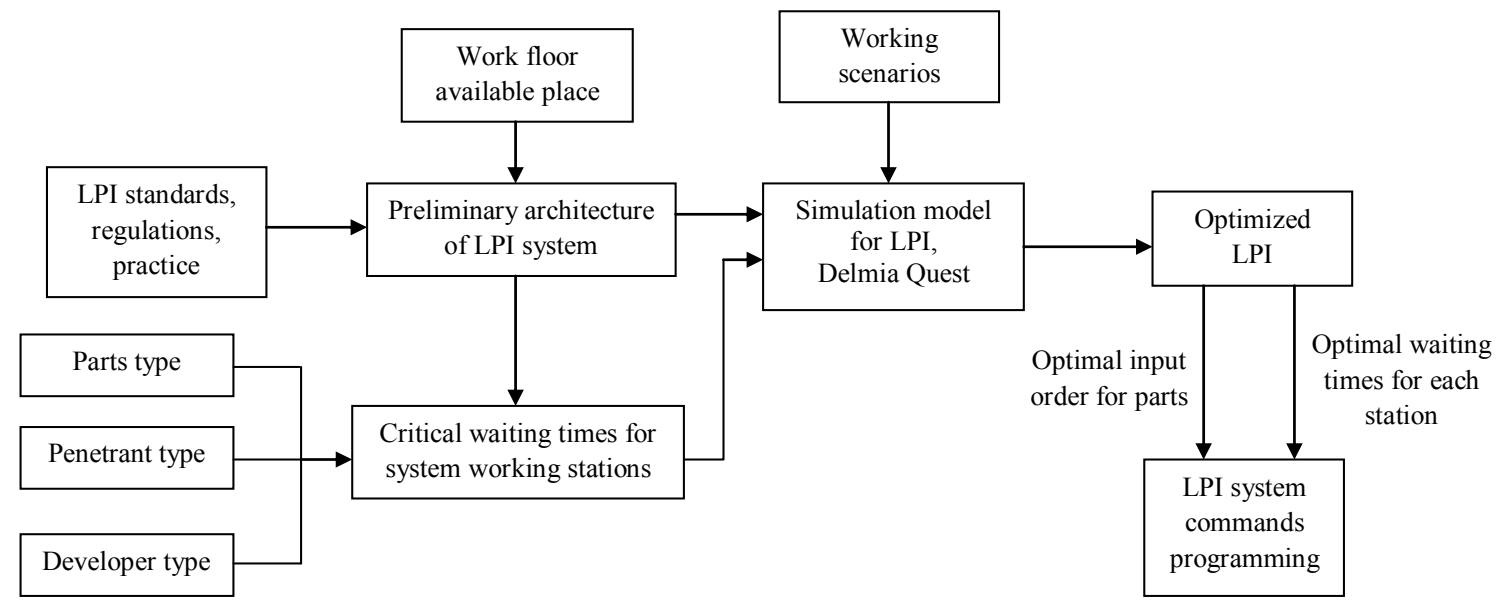

Figure 3: Research approach for optimizing a LPI system.

The research approach used in the paper is presented in Fig. 3 and detailed below:

1. Establishing the preliminary architecture of the LPI system.

2. Establishing the process times for each type of inspected part and determining the critical times within the model which need optimization. 
3. Building a simulation model based on the preliminary architecture and assumptions.

4. Running the simulation model in different scenarios in which the critical times are distributed differently between WS and the input order of inspected parts differs.

5. Optimizing the system based on the analysis of the simulation results.

\section{LPI SYSTEM SIMULATION MODEL}

The developed simulation model (Fig. 4) is based on the following assumptions:

- There is an 8 hours shift, there are no breaks during the shift.

- The parts are loaded in the system from the buffers in an order which repeats during the whole shift.

- The operation time for each working station is different for each part family.

- The working stations are equally distributed along the conveyor length.

- The waiting times are fixed, but they can be distributed between different working stations.

- In each working station only one part is loaded at one time.

- Machines and part transporter line are available full time, with no breakdowns.

The automated LPI system was modelled using a discrete simulation method type push-in. The system model has as structural elements: five working stations (WS2-WS6), one conveyor and three buffers for parts loading. For the LPI system, the parts transfer from the buffers to the conveyor is made using specific automated devices which are not represented in the model as separate entities. The order of loading parts in the system is made by the order of sources link. In the model for each type of parts in each working station were created specific processes (type: constant time). For example, for inspection, the following working stations were created: $i n s p_{-} 1$ for parts type 1 ; insp_2 for parts type 2 and insp_3 for parts type 3 .

The input parameters for the simulations are the working times in each station, waiting times and transportation times. The system configuration is maintained identical in all the simulations run. The parts are drying not only in WS4 (using hot air), but also during the transportation time. Therefore, in order to control the drying time, due to the fact that it is difficult to give a fair estimation of the time equivalence between drying the parts in open air and drying the parts under hot air, we maintained constant the time corresponding to WS4 during all the simulations and during the real inspection process.

The optimisation of the system consisted in time distribution between working stations in order to decrease the idle time and the block time of each WS. Thus, the formulas (1) - (5) describe the initial working condition for the system simulation. These formulas were established based on LPI process times (presented in Table I). For optimising the productivity of the LPI system, a new set of formulas were developed (6) - (12) in which the transportation and waiting times are distributed between workstations.

The following notations are used in the formulas (1) - (12):

$T_{p e n} \quad$ - penetrant spraying time

$T_{w} \quad-$ penetrant washing time

$T_{D w} \quad-$ dwell time

$T_{D T} \quad$ - drying time

$T_{D} \quad$ - developer spraying time

$T_{D v} \quad$ - developer drying time

$T_{\text {insp }} \quad$ - automate inspection time

$T_{\text {tr } 23} ; T_{\text {tr } 34} ; \operatorname{Trt}_{45} ; T_{\text {tr } 56}$ - transportation time between stations

(e.g. $T_{t r 23}$ represents the transportation time between WS2 and WS3)

$T_{W S i} \quad$ process time for parts in each WS, where $i=2 \ldots 6$

Two scenarios are considered in the research, as detailed bellow: 
Scenario 1 - In the first scenario the waiting time and transportation time were allocated to WS3 and WS5 (the drying time in WS4 was maintained unchanged from the reason explained before). Thus, for each WS and for each part $P 1, P 2, P 3$ (see also the values in Table I), the corresponding process times $T_{W S i}$ are calculated as follows:

$$
\begin{gathered}
T_{W S 2}=T_{p e n} \\
T_{W S 3}=T_{D w}+T_{t r 23}+T_{w}+T_{t r 34} \\
T_{W S 4 p}=T_{D T} \\
T_{W S 5}=T_{t r 45}+T_{D}+T_{D v}+T_{t r 56} \\
T_{W S 6}=T_{\text {insp }}
\end{gathered}
$$

Scenario 2 - In the second scenario, the transportation time was allocated to WS2, WS3, WS5 and WS6, while the waiting times were divided between WS3 and WS5 in different ratios expressed using $\alpha, \beta, \gamma$ and $\delta$ constants as presented in Table II, so that eqs. (11) and (12) are satisfied.

$$
\begin{gathered}
T_{W S 2}=T_{p e n}+\alpha * T_{D w}+T_{t r 23} \\
T_{W S 3}=\beta * T_{D w}+T_{w}+T_{t r 34} \\
T_{W S 4 p}=T_{D T} \\
T_{W S 5}=T_{t r 45}+T_{D}+\gamma * T_{D v} \\
T_{W S 6}=T_{t r 56}+\delta * T_{D v}+T_{\text {insp }} \\
\alpha+\beta=1 \\
\gamma+\delta=1
\end{gathered}
$$

Comparing to scenario 1 in which the penetrant is dried only in WS3, in scenario 2 the time required for drying the penetrant, $T_{D w}$, is divided between WS2 and WS3 (Table II), so that, as an example, $10 \%$ (i.e. $\alpha=0.1$ ) of the time is allocated to WS2 (see (6)), while $90 \%$ (i.e. $\beta=0.9$ ) of the time is allocated to WS3 (see (7)); the time distribution being referred as $10-90 \%$.

Table II: Constants showing distributions of waiting and transportation times between WS.

\begin{tabular}{|l|l|l|l|l|l|l|l|l|l|}
\hline$\alpha, \gamma$ & 0.10 & 0.20 & 0.30 & 0.40 & 0.50 & 0.60 & 0.70 & 0.80 & 0.90 \\
\hline$\beta, \delta$ & 0.90 & 0.80 & 0.70 & 0.60 & 0.50 & 0.40 & 0.30 & 0.20 & 0.10 \\
\hline
\end{tabular}

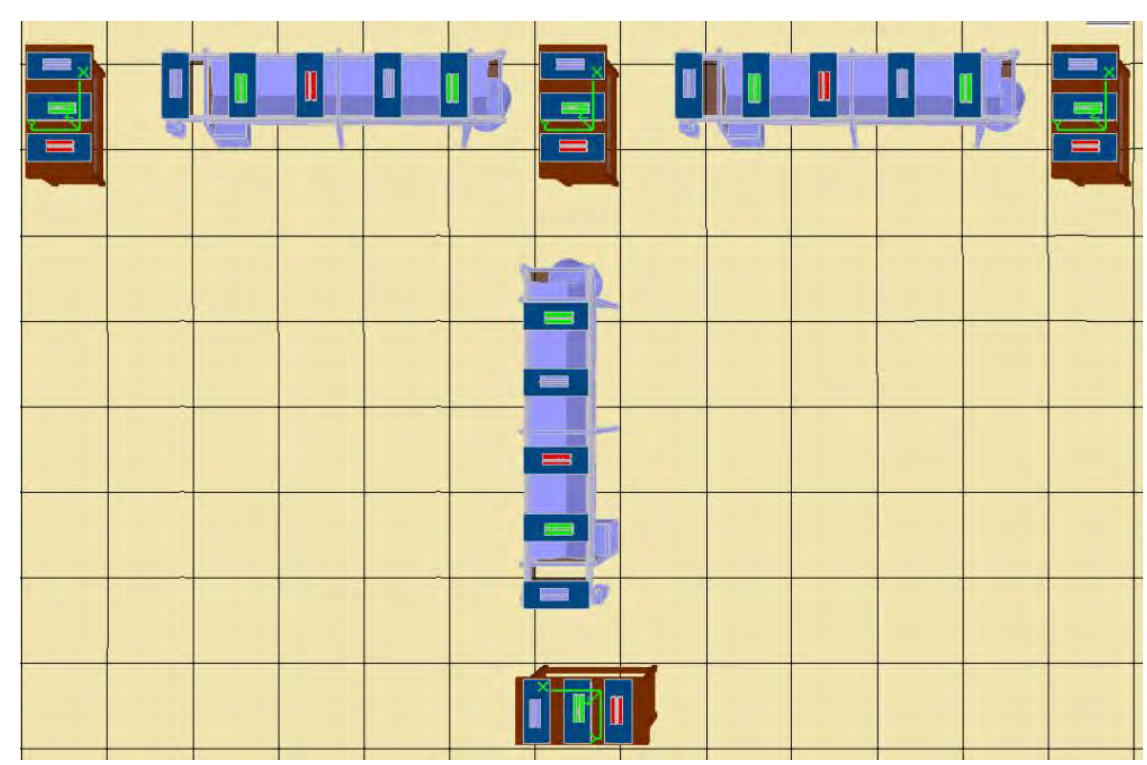

Figure 5: LPI system model in Delmia Quest. 


\section{OPTIMIZED AUTOMATED LPI SYSTEM}

Two analyses were performed for each scenario. The first analysis was made for the case in which only parts from one family are inspected by the system during a shift, i.e. first only $P 1$ are inspected in 8 hours, then only $P 2$ are inspected in the next 8 hours, etc. The second analysis was made for different combinations in which the three types of parts are inspected (e.g. 1-2-3; 1-3-2; 2-3-1; 2-1-3; 3-2-1; 3-1-2).

The first analysis, for each of the two scenarios presented in section 3 , was made for analysing the productivity of the system if the same type of part is inspected during the shifts.

In scenario 1, simulations were run using the parameters from Table I which correspond to the current approach used in the manual liquid penetrant inspection process.

In scenario 2, the waiting times and transportation times were distributed (in different percentage between working stations, as presented in Table II and eqs. (6) - (12)). Table III presents the input parameters in the simulation for 50-50\% distribution of the waiting and transportation times. Several simulations were run to determine the effect on productivity of various distribution ratios of the waiting time and transportation time between stations. The conclusion after running the simulations was that the optimal results, for the first analysis, are obtained in scenario 2 , for $50-50 \%$ distribution of waiting time and transportation time between working stations.

Table III: Input parameters, $50-50 \%$ distribution of waiting and transportation times.

\begin{tabular}{|c|l|c|c|c|}
\hline WS & \multicolumn{1}{|c|}{ Time structure } & $\boldsymbol{P 1}$ & $\boldsymbol{P 2}$ & $\boldsymbol{P 3}$ \\
\hline \multirow{2}{*}{ WS2 } & Penetrant spraying & $10 \mathrm{~s}$ & $10 \mathrm{~s}$ & $10 \mathrm{~s}$ \\
& Waiting time + transportation time & $150 \mathrm{~s}$ & $150 \mathrm{~s}$ & $150 \mathrm{~s}$ \\
\hline \multirow{2}{*}{ WS3 } & Waiting time + transportation time & $150 \mathrm{~s}$ & $150 \mathrm{~s}$ & $150 \mathrm{~s}$ \\
& Penetrant washing & $10 \mathrm{~s}$ & $15 \mathrm{~s}$ & $20 \mathrm{~s}$ \\
\hline WS4 & Drying & $200 \mathrm{~s}$ & $250 \mathrm{~s}$ & $350 \mathrm{~s}$ \\
\hline \multirow{2}{*}{ WS5 } & Developer spraying & $7 \mathrm{~s}$ & $10 \mathrm{~s}$ & $12 \mathrm{~s}$ \\
& Waiting time + transportation time & $300 \mathrm{~s}$ & $250 \mathrm{~s}$ & $200 \mathrm{~s}$ \\
\hline \multirow{2}{*}{ WS6 } & Inspection & $300 \mathrm{~s}$ & $250 \mathrm{~s}$ & $200 \mathrm{~s}$ \\
& Waiting time +transportation time & $40 \mathrm{~s}$ & $30 \mathrm{~s}$ & $50 \mathrm{~s}$ \\
\hline
\end{tabular}

Table IV presents a comparison of the simulations results for the scenario 1 (see parameters from Table I and eqs. (1) - (5)) and the results in scenario 2 for $50-50 \%$ ratio (see parameters from Table III and eqs. (6) - (12)). One can notice that the number of parts is increasing and also the block time for WS2 and WS4 is decreasing.

In the second analysis, the system productivity was analysed to see if it is increasing by combining the input order of the 3 types of parts in the system.

In scenario 1, the simulation input parameters were calculated based on parameters from Table I and eqs. (1) - (5). The system productivity resulted from the simulations is presented in Table $\mathrm{V}$ and it one can notice that the same number of parts is inspected in the system, regardless the order in which the parts are pulled from the buffers.

In scenario 2, different distribution ratios were simulated for different parts' loading combinations, the system productivity being presented in Table VI for 1-2-3 loading order, while in Table VII is shown the blockage of each working stations for the same loading order, as an example. The distribution 50-50\% was also proved optimal for increasing the productivity and for decreasing the idle times and blocking time in the system. 
Table IV: System productivity for analysis, comparison Scenario 1 vs. Scenario 2 (50-50 \%).

\begin{tabular}{|c|l|c|c|c|c|c|c|}
\hline \multirow{2}{*}{ No. of parts } & $\begin{array}{c}\text { Scenario } \\
\mathbf{1}\end{array}$ & $\begin{array}{c}\text { Scenario } \\
\mathbf{2}\end{array}$ & $\begin{array}{c}\text { Scenario } \\
\mathbf{1}\end{array}$ & $\begin{array}{c}\text { Scenario } \\
\mathbf{2}\end{array}$ & $\begin{array}{c}\text { Scenario } \\
\mathbf{1}\end{array}$ & $\begin{array}{c}\text { Scenario } \\
\mathbf{2}\end{array}$ \\
\cline { 3 - 8 } & $\mathbf{P 1 - 4 6}$ & $\mathbf{P 1 - 8 2}$ & $\mathbf{P 2 - 5 2}$ & $\boldsymbol{P 2 - 9 9}$ & $\boldsymbol{P 3 - 6 8}$ & $\boldsymbol{P 3 - 8 0}$ \\
\hline \multirow{3}{*}{ WS2 } & idle & 0 & 0 & 0 & 0 & 0 & 0 \\
\cline { 2 - 8 } & busy & 1.7 & 91.8 & 2 & 93.7 & 2.5 & 61.3 \\
\cline { 2 - 8 } & blocked & 98.3 & 8.2 & 98 & 6.3 & 97.5 & 38.7 \\
\hline \multirow{3}{*}{ WS3 } & idle & 0.1 & 5.9 & 0 & 3.4 & 0.1 & 0.9 \\
\cline { 2 - 8 } & busy & 52.7 & 54.8 & 63 & 64.4 & 78.1 & 48.6 \\
\cline { 2 - 8 } & blocked & 47.2 & 39.4 & 37 & 32.2 & 21.8 & 50.5 \\
\hline \multirow{3}{*}{ WS4 } & idle & 1.5 & 4.2 & 1.4 & 1.7 & 1.1 & 1.3 \\
\cline { 2 - 8 } & busy & 33.3 & 58.5 & 49.2 & 88.4 & 84.2 & 98.7 \\
\cline { 2 - 8 } & blocked & 65.2 & 37.3 & 49.4 & 9.8 & 14.6 & 0 \\
\hline \multirow{3}{*}{ WS5 } & idle & 1.8 & 3.4 & 2 & 3.2 & 2.4 & 55.3 \\
\cline { 2 - 8 } & busy & 98.2 & 53.5 & 98 & 61.4 & 97.6 & 44.7 \\
\cline { 2 - 8 } & blocked & 0 & 43.1 & 0 & 35.5 & 0 & 0 \\
\hline \multirow{3}{*}{ WS6 } & idle & 93.6 & 3 & 94.3 & 3 & 8.2 & 30.6 \\
\cline { 2 - 8 } & busy & 6.4 & 97 & 5.7 & 97 & 11.8 & 69.4 \\
\cline { 2 - 8 } & blocked & 0 & 0 & 0 & 0 & 0 & 0 \\
\hline
\end{tabular}

When summarizing the simulation study results analysis, the maximal value of the productivity of the LPI system is obtained when the critical waiting times within the systems (corresponding to dwell, penetrant and developer operations) are distributed 50-50\% between WS, in the case the parts are pulled from the buffers and inspected in the order P1-P2-P3.

Table V: System productivity in the analysis 2 - scenario 1.

\begin{tabular}{|c|c|c|c|c|c|c|}
\hline Parts loading order & $\mathbf{1 - 2 - 3}$ & $\mathbf{1 - 3 - 2}$ & $\mathbf{2 - 1 - 3}$ & $\mathbf{2 - 3 - 1}$ & $\mathbf{3 - 1 - 2}$ & $\mathbf{3 - 2 - 1}$ \\
\hline $\boldsymbol{P 1}$ & 19 & 19 & 18 & 18 & 18 & 18 \\
\hline $\boldsymbol{P 2}$ & 18 & 18 & 19 & 19 & 18 & 18 \\
\hline $\boldsymbol{P 3}$ & 18 & 18 & 18 & 18 & 19 & 19 \\
\hline Total & 55 & 55 & 55 & 55 & 55 & 55 \\
\hline
\end{tabular}

Table VI: System productivity for different ratios of waiting and transportation times distribution for analysis 2 - scenario 2 , loading order 1-2-3.

\begin{tabular}{|c|c|c|c|c|}
\hline 1-2-3 loading order & P1 & P2 & P3 & Total \\
\hline$\alpha=10 \%, \beta=90 \%, \gamma=10 \%, \delta=90 \%$ & 19 & 19 & 19 & 57 \\
\hline$\alpha=20 \%, \beta=80 \%, \gamma=20 \%, \delta=80 \%$ & 21 & 21 & 21 & 63 \\
\hline$\alpha=30 \%, \beta=70 \%, \gamma=30 \%, \delta=70 \%$ & 24 & 24 & 23 & 71 \\
\hline$\alpha=40 \%, \beta=60 \%, \gamma=40 \%, \delta=60 \%$ & 28 & 27 & 27 & 82 \\
\hline$\alpha=50 \%, \beta=50 \%, \gamma=50 \%, \delta=50 \%$ & 32 & 32 & 32 & 96 \\
\hline$\alpha=60 \%, \beta=40 \%, \gamma=60 \%, \delta=40 \%$ & 30 & 30 & 30 & 90 \\
\hline$\alpha=70 \%, \beta=30 \%, \gamma=70 \%, \delta=30 \%$ & 26 & 26 & 25 & 77 \\
\hline$\alpha=80 \%, \beta=20 \%, \gamma=80 \%, \delta=20 \%$ & 23 & 23 & 22 & 68 \\
\hline$\alpha=90 \%, \beta=10 \%, \gamma=90 \%, \delta=10 \%$ & 21 & 20 & 20 & 61 \\
\hline
\end{tabular}


Table VII: Simulation results for analysis 2 - scenario 2, 1-2-3 order, 50-50 \% time allocation.

\begin{tabular}{|l|l|c|c|c|c|c|}
\hline Productivity & & WS2 & WS3 & WS4 & WS5 & WS6 \\
\cline { 2 - 7 } (no. of parts): & idle & 0 & 8.2 & 1.9 & 4.8 & 3.1 \\
\cline { 2 - 7 } P1: 32, P2: 32, P3: 32 & busy & 90.4 & 61.4 & 90.7 & 58.7 & 96.9 \\
\cline { 2 - 7 } 1-2-3 loading order & blocked & 9.6 & 30.4 & 7.4 & 36.6 & 0 \\
\hline
\end{tabular}

The simulation results were used for programming the system, thus avoiding running a large number of tests. Fig. 6 presents an excerpt from the programming code where the simulation data were introduced.

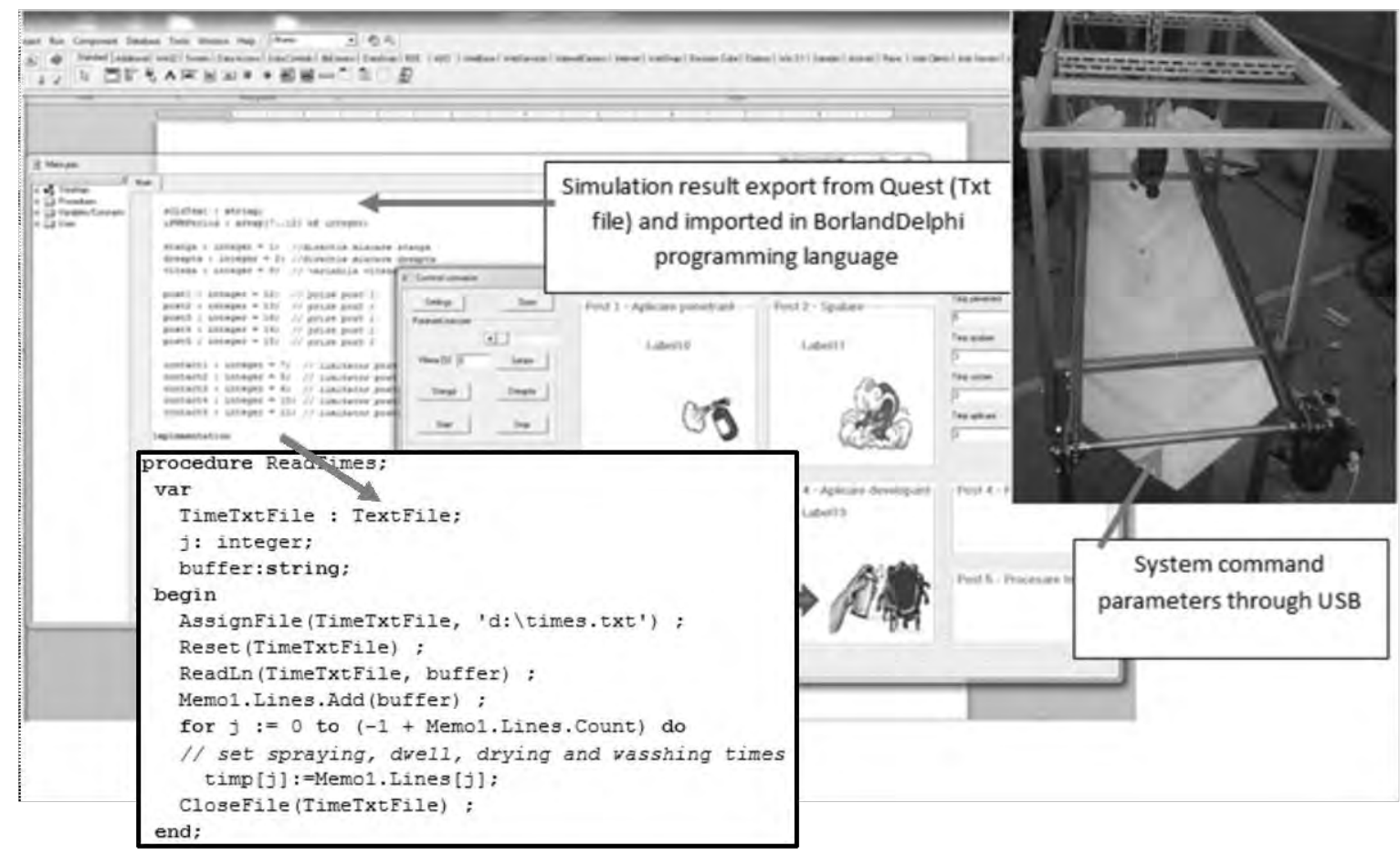

Figure 6: Programming code excerpt for LPI system containing data from the simulation.

Table VIII: System productivity for analysis 2 - scenario 2, 50-50 \% time allocation.

\begin{tabular}{|c|c|c|c|c|c|c|}
\hline Part & $\mathbf{1 - 2 - 3}$ & $\mathbf{1 - 3 - 2}$ & $\mathbf{2 - 1 - 3}$ & $\mathbf{2 - 3 - 1}$ & $\mathbf{3 - 1 - 2}$ & $\mathbf{3 - 2 - 1}$ \\
\hline $\boldsymbol{P 1}$ & 32 & 31 & 31 & 30 & 30 & 31 \\
\hline $\boldsymbol{P 2}$ & 32 & 31 & 31 & 30 & 30 & 31 \\
\hline $\boldsymbol{P 3}$ & 32 & 31 & 30 & 30 & 30 & 30 \\
\hline Total & 96 & 93 & 92 & 90 & 90 & 92 \\
\hline
\end{tabular}

\section{CONCLUSIONS}

Manufacturing quality products is mandatory for producers in obtaining and maintaining a competitive position on a highly demanding market. Therefore the importance of continuously improving manufacturing processes [28] - on one hand, and inspecting and evaluating each part quality, on the other hand. In this context, this paper proposes an increasing productivity simulation oriented algorithm developed in Delmia Quest for a new fully-automated LPI system case study. The new LPI system considered here is used in the inspection process of different types of parts for the open discontinuities on the parts' surface 
evaluation and accordingly giving the passing/fail verdict. The authors consider that a detailed analysis of these systems is beyond the purpose of this paper, the focus being on optimizing the new LPI system using discrete material flow simulation. Therefore only brief characteristics are mentioned, with the aim to emphasize the similarity with other systems that however are not fully automated, nor were their optimization analysed/presented in the literature. Nevertheless the authors intend to carefully focus on the technical performances of each module of this system in future research.

The purpose of this case study was related to the need to develop the command/control software for the new system and therefore to establish the values of some functional parameters (such as starting and ending times of the spraying process) without performing too many experimental tests which are resources consuming. Two analyses were made. A first analysis considered the case when only one type of part is inspected during the entire simulation time, while in the second analysis different combinations of parts belonging to each family were submitted to the inspection process during the simulation time. In each analysis, two scenarios were considered. For the first analysis in the first scenario (initial parameters from Table I), the simulations results showed that during the 8 hours shift $P 1-46$ parts, $P 2-55$ parts, $P 3-68$ parts can be inspected, while in the optimized case (scenario 2, input parameters from Table II in 50-50 \% time allocation) $P 1-82$ parts, $P 2-99$ parts, $P 3-$ 80 parts can be inspected.

In the second analysis, two scenarios were run as well. In scenario 1, the input parameters used in the model were the operation times for each working stations as obtained by practice, type of part/defect and according to the liquid penetrant inspection standards and regulations. The simulations results showed that in scenario 1 (input data from Table I) regardless the parts order, the productivity of the system is the same: totally 55 parts in 8 hours shift. In scenario 2, the waiting times for the working stations were optimized and the simulations results in 50-50\% time allocation showed an increase of the productivity to $90-96$ parts, depending on the loading order. Based on these results it was concluded that the second scenario of the second analysis is the best solution for the LPI system productivity.

The research presented in the paper showed that simulation data can be used with success for programming the LPI system by determining the optimal values for different functional parameters. Moreover, the authors consider that a similar approach can be used for designing and optimizing other in line automated manufacturing systems, contributing to a significant decrease of the number of tests which are time and money consuming.

Further research will be focused on performing tests on the LPI system and on developing the image processing software for detecting the parts defects.

\section{ACKNOWLEDGEMENT}

This work has been co-funded by the Sectoral Operational Programme Human Resources Development 2007-2013 of the Romanian Ministry of Labour, Family and Social Protection through the Financial Agreement POSDRU/89/1.5/S/62557.

\section{REFERENCES}

[1] Shull, P. J. (2002). Nondestructive Evaluation - Theory, Techniques, and Applications, CRC Press (Marcel Dekker), New York, Ch. 2, 17-56, doi:10.1201/9780203911068

[2] Hellier, C. J. (2001). Handbook of Nondestructive Evaluation, Mc-Graw Hill, Boston, 2.1-27, 4.1-32

[3] $P N-E N 473$ (2002). Non-destructive tests. Qualification and certification of personnel responsible for non-destructive tests. General rules. 
[4] SR EN 571-1 (1999). Non-destructive examination. Liquid penetrant inspection. Part 1: General rules.

[5] SR EN 10228-2 (2000). Non-destructive testing of steel forgings. Part 2: Penetrant testing.

[6] ASTM E 16 (1995). Standard Test Method for Liquid Penetrant Examination.

[7] EN ISO 5817 (2004). Welding - fusion-welded joints in steel, nickel, titanium and their alloys (beam welding excluded). Quality levels for imperfections.

[8] DIN EN 1330-6 (1994). Non-destructive Testing - Terminology - Part 6: Terms Used In Penetrant System.

[9] Law, A. M.; Kelton, W. D. (2000). Simulation, modelling \& analysis, $3^{\text {rd }}$ Edition, McGraw-Hill, Boston

[10] Robinson, S. (2003). Simulation - The Practice of Model Development and Use, John Wiley \& Sons, Sussex, 63-93

[11] Gill, A. (2008). Identifying potential bottlenecks through activity under-utilization cost, International Journal of Simulation Modelling, Vol. 7, No. 4, 165-175, doi:10.2507/IJSIMM07(4)1.104

[12] Banks, J.; Carson, J.; Nelson, B.; Nicol, D. (2009). Discrete-Event System Simulation, $5^{\text {th }}$ edition, Prentice Hall, 1-17, 55-70

[13] Masood, S. (2006). Line balancing and simulation of an automated production transfer line, Assembly Automation, Vol. 26, No. 1, 69-74, doi:10.1108/01445150610645684

[14] Arisha, A.; Young, P.; El Baradie, M. (2003). Evaluation of Scheduling Strategies on the Performance of a Flexible Manufacturing Cell - A Simulation Study, Proceedings of the $32^{\text {nd }}$ International Conference on Computers and Industrial Engineering, Limerick, Ref. 280, 700-706

[15] Longo, F.; Mirabelli, G.; Papoff, E. (2006). Effective design of an assembly line using modeling \& simulation, Proceedings of the 2006 IEEE Winter Simulation Conference, 1893-1898, doi:10.1109/WSC.2006.322971

[16] Garcia-Chamizo, J. M.; Fuster-Guillo, A.; Azorin-Lopez, J. (2007). Simulation of Automated Visual Inspection Systems for Specular Surfaces Quality Control, Advances in Image and Video Technology, Lecture Notes in Computer Science, Vol. 4872, 749-762, doi:10.1007/978-3-54077129-6_64

[17] Bzymek, Z. M.; Nunez, M.; Li, M.; Powers, S. (2008). Simulation of a machining sequence using Delmia/Quest software. Computer-Aided Design and Applications, Vol. 5, No. 1-4, 401-411, doi:10.3722/cadaps.2008.401-411

[18] Ekren, B. Y.; Ornek, A. M. (2008). A simulation based experimental design to analyze factors affecting production flow time, Simulation Modelling Practice and Theory, Vol. 16, No. 3, 278 293, doi:10.1016/j.simpat.2007.11.016

[19] Nguyen, D. M. (2009). Empirical manufacturing line designs in Japanese automobile plants, International Journal of Simulation Modelling, Vol. 8, No. 2, 69-80, doi:10.2507/IJSIMM08(2) 1.119

[20] Vetterlein, T.; Wagener, M.; Rongen, H.; Sampson, C. (2006). Automated dye penetrant systems with process control and documentation in the aerospace industry, Insight - Non-Destructive Testing and Condition Monitoring, Vol. 48, No. 3, 171-173, doi:10.1784/insi.2006.48.3.171

[21] http://www.johnsonandallen.co.uk/dpi-products.htm, accessed on 15-12-2011

[22] http://www.arcspecialties.com/projectdetails.aspx?projid=3, accessed on 15-12-2011

[23] http://www.ramkleen.com/case_studies/fpi2.html, accessed on 15-12-2011

[24] http://www.testron.ru/eng/catalog/C42/, accessed on 18-01-2012

[25] http://www.proceco.com, accessed on 18-01-2012

[26] US Patent 3762216 (1973). Automated liquid penetrant inspection system.

[27] US Patent 7215807 (2007). Non-destructive inspection method and apparatus.

[28] Wang, Y.-C.; Chen, C.-H.; Lee, B.-Y.; Liang, T.-H.; Chung, T.-S. (2012). A measurement system for the fabrication inspection of linear and rotary axes in a 5-axis tool grinder, Technical Gazette, Vol. 19, No. 3, 583-587 